\title{
10
}

\section{Surge Modeling in Sewers using Alternative Hydraulic Software Programs}

\section{Karen E. Ridgway and Gregory Kumpula}

Rapid filling, such as from large and intense rain storms, can result in the formation of open channel surge waves (hydraulic bores) with steep wave fronts in sewers/tunnels. Hydraulic bores travel in the sewers/tunnels until they reach an upstream, downstream or internal boundary (e.g. at shafts or manholes). As the sewer/tunnel system fills, the bores wholly or partially reflect off the boundaries and discontinuities of the sewer/tunnel system causing surges in hydraulic grade line (HGL) elevations that rise above ground surface elevations. Surges are important to evaluate and control in deep relief sewers and tunnels since they can cause high internal pressures that can stress the conduit walls, damage manhole/shaft structures, and cause spilling of combined sewage to the environment. Stress reversals can loosen structures and their anchors.

It is important that the hydraulic modeling software used for the design of the sewer/tunnel facility accurately compute surge conditions since the cost for surge control measures can be significant, and failures due to improperly controlled surges can be catastrophic. The required surge control measures depend on the proposed operational scheme, and the control measures may be over- or under-sized depending on the hydraulic software program used for the design.

Ridgway, K. and G.J. Kumpula. 2008. "Surge Modeling in Sewers using Alternative Hydraulic Software Programs." Journal of Water Management Modeling R228-10. doi: 10.14796/JWMM.R228-10. (C) CHI 2008 www.chijournal.org ISSN: 2292-6062 (Formerly in Reliable Modeling of Urban Water Systems. ISBN: 978-0-9808853-0-9) 
Hydraulic modeling software programs currently in common use for sewer system hydraulic modeling were not developed to simulate rapidly varying open channel transient conditions. These programs, as commonly applied to sewer systems, will under predict and over damp the surge waves.

\subsection{Physical Model}

Physical model data obtained from the University of Michigan hydraulics laboratory were used to evaluate the hydraulic model's capability in predicting surge. This physical model was built in order to demonstrate rapid filling scenarios in deep underground storage tunnels. Hydraulic model simulations were constructed in order to simulate the physical model conditions as closely as possible. By comparing physically measured parameters such as HGL to the hydraulic model simulation results, the ability of the software programs to simulate surges can be judged.

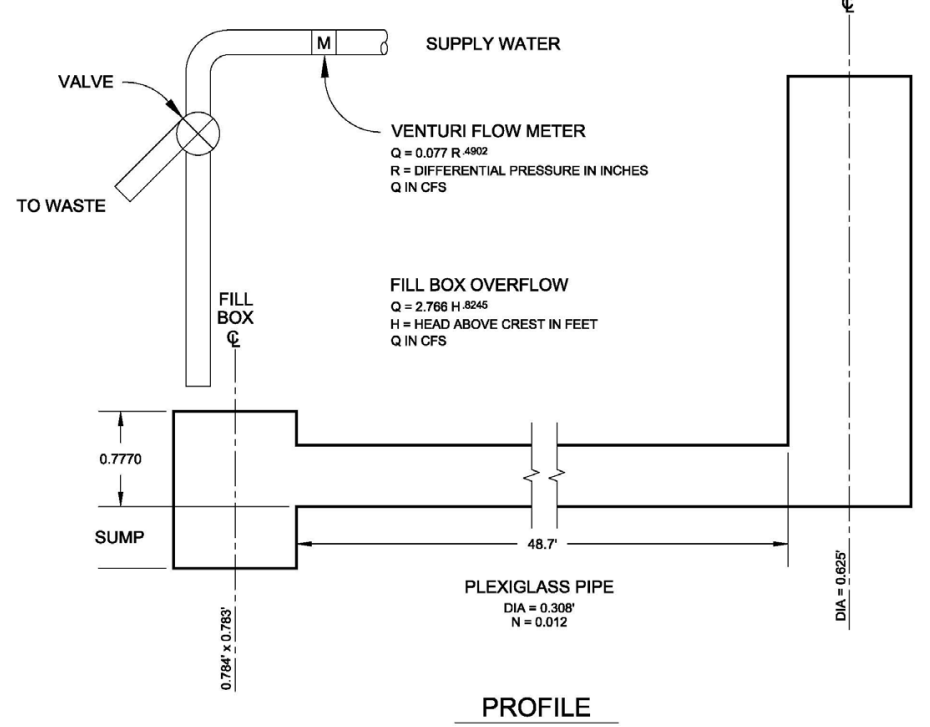

Figure 10.1 Physical model configuration.

The physical model is a single $48.7 \mathrm{ft}(14.8 \mathrm{~m})$ long, 4 in. $(102 \mathrm{~mm})$ diameter pipe with a fill box at one end and a riser shaft at the other end, shown in Figure 10.1. The pipe was tested at a level condition and sloping 
slightly in both directions. The pipe was tested with varying levels of initial water depth at rest. The physical model experiments are fully presented in the listed reference (Vasconcelos 2005).

A high steady filling rate is suddenly put into the fill box, and a hydraulic bore is created, rapidly filling the pipe from the fill box to the riser shaft, and then reflecting back and forth between each end. The relative minimum and maximum HGL was recorded at the riser shaft for comparison to the hydraulic model simulations.

\subsection{Compared Programs}

Hydraulic modeling software programs used in this comparison were limited to those programs that are able to simulate the physical geometry and flow conditions experienced in the physical models. Because these models will produce a solution, it can be assumed that they will be used for surge modeling whether or not the results are accurate. This concern shows the need for a comparison of results among the programs.

The one-dimensional hydraulic software programs used in this comparison include SWMM5 by the US EPA, MOUSE by DHI, InfoWorks by Wallingford Software, and TAP by ASI. All of these programs utilize a Preissmann slot above the conduit crown to track HGL when surcharged conditions occur (Preissmann 1961). However, the slot width in TAP is calculated from the user specified closed conduit wave speed and can be very small and provide insignificant amounts of storage volume.

TAP is a hydraulic modeling program that solves the fully dynamic onedimensional equations of continuity and momentum using a finite volume solution method. TAP simulates discontinuities in the hydraulic grade line (HGL) including open channel and pressure wave fronts using Roe's first order upwind scheme with an approximate Riemann solver (Roe 1981).

TAP models include "links" that may be channels/conduits, gates, dams, or pumps. TAP requires that the "links" have junctions at each end. The channels/conduits in the system being analyzed are divided into uniformlyspaced small cells. Time steps are automatically calculated and selected to be stable and can vary throughout the model simulation. TAP employs a local time stepping scheme (Crossley 2003). For each cell and for each time step, the fully dynamic equations are solved, and cell averaged quantities of depth, area, velocity, and flow rates are provided. More than one channel/conduit may be connected to any junction, and the system being modeled may be branched or looped. 
The development of TAP is more fully discussed in the listed reference (Ridgway and Kumpula 2006).

The model results were compared to each other and the physical model, and possible explanations for discrepancies are discussed.

\subsection{Comparison Results}

The physical model results were videotaped and tabulated at the riser shaft. One model setup was selected for evaluation. This setup has the pipe sloping from the riser to the fill box with the surge wave traveling up slope and an initial water depth in the pipe at about two-thirds full. For this setup, no significant air pockets were trapped by the surge wave.

As the fill box received a large steady flow rate, a hydraulic bore was created that rapidly filled the pipe and ran to and reflected off the riser shaft. While the HGL created at the fill box never exceeded $0.82 \mathrm{ft}$ of water depth, the momentum of the bore created a peak HGL at the riser shaft that reached $1.60 \mathrm{ft}$, nearly double that of the driving head.

Further peak and low HGL values and times were tabulated for the first 70 seconds of the physical model run. These peaks and lows are presented in Table 10.1.

Table 10.1 Physical model results.

\begin{tabular}{lll}
\hline Time $(\mathrm{sec})$ & Riser HGL (feet) & Reading Type \\
\hline 14.0 & 1.60 & Maximum \\
29.3 & 1.22 & Maximum \\
37.0 & 0.45 & Minimum \\
45.3 & 1.08 & Maximum \\
53.0 & 0.58 & Minimum \\
61.0 & 1.00 & Maximum \\
69.0 & 0.66 & Minimum \\
\hline
\end{tabular}

\subsubsection{TAP Results}

The creation of the TAP model was simple and straightforward. Because TAP is designed to simulate surge conditions accurately, the optimized time step is automatically calculated and used for each conduit in a network. The 
conduit was automatically divided into an appropriate number of "cells" and the hydraulic bore was tracked accurately.

Using the TAP model setup, an initial surge wave peak HGL of $1.54 \mathrm{ft}$, close to the $1.60 \mathrm{ft}$ measured in the physical model. Additionally, later peak HGL results remain in close alignment with the physical model, demonstrating the ability of TAP to also simulate the damping experienced in the system. Figure 10.2 shows the close relationship between the TAP simulation results and the physical model measurements.

The TAP model setup that provided good comparisons had a cell size equal to the pipe diameter. Finer cells would provide essentially the same answers but would require longer computational time. The TAP model therefore had 158 cells that were each about 4 in. in length. The open channel wave speed in TAP was calculated for each cell at each time step based on the water depth and the shallow water wave equation. The surge calculations were not strongly dependent on the closed conduit wave speed for the simulation and a closed conduit wave speed of $400 \mathrm{ft} / \mathrm{s}$ was utilized.

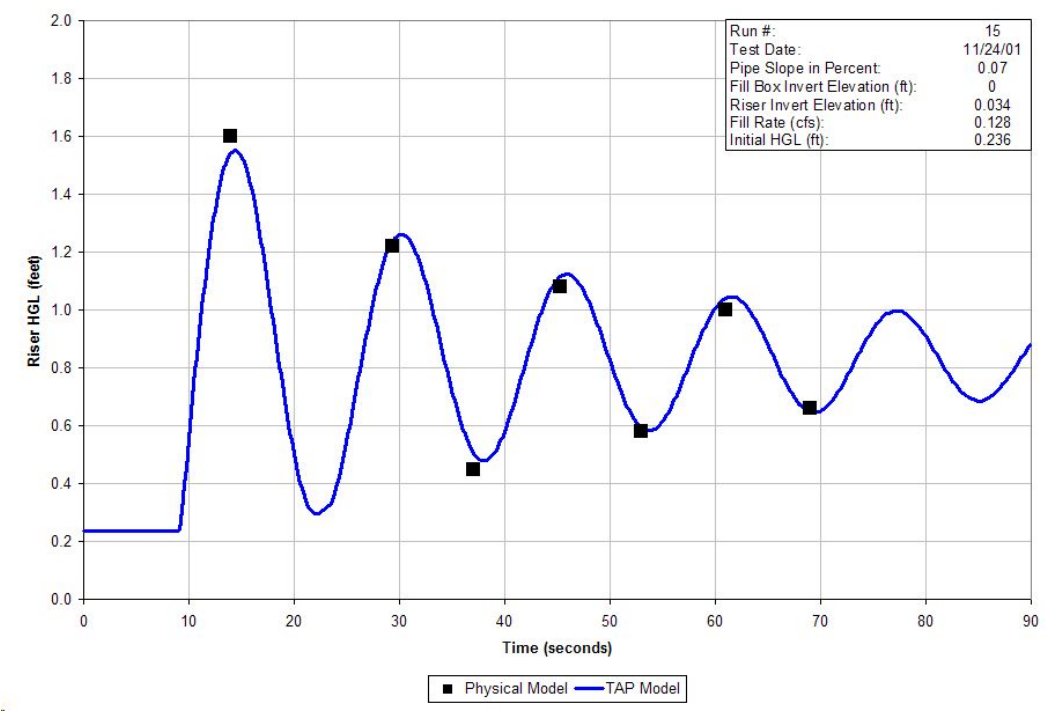

Figure 10.2 Comparison between physical model measurements and the TAP model simulation results. 


\subsubsection{SWMM5 Results}

The initial setup of the SWMM5 model was completed using a single conduit to model the 4 in. diameter pipe. Storage nodes were used to simulate both the fill box and the riser shaft, and a weir was used to model the overflow at the fill box.

Using this initial setup produced an initial surge wave peak HGL of only $0.92 \mathrm{ft}$, well below the physical model result of $1.60 \mathrm{ft}$. Not only was this peak HGL low, but it also occurred $48 \mathrm{sec}$ after the large inflow at the fill box was introduced, well after the $14 \mathrm{sec}$. delay shown in the physical model. A comparison of these SWMM5 model results alongside the physical model results are shown in Figure 10.3.

The main reason for the poor comparison is that the surge wave front is numerically averaged across the single length of pipe during each time step in the SWMM5 model.

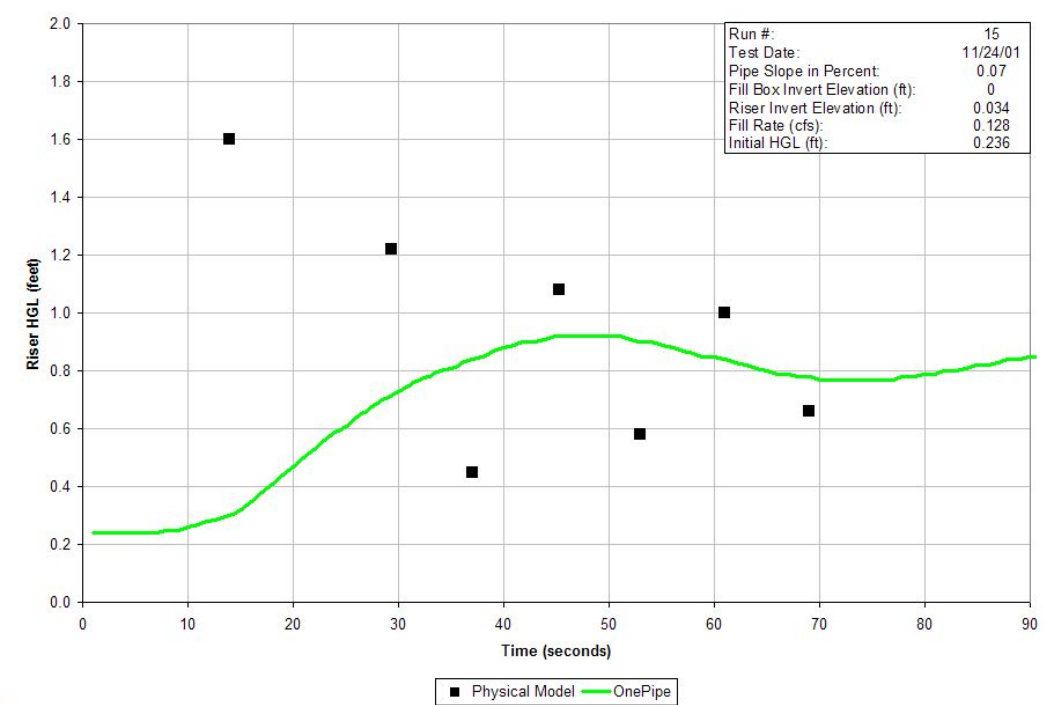

Figure 10.3 Comparison between physical model measurements and the SWMM5 model simulation results.

Further investigation showed that it is possible to achieve more accurate results using SWMM5 by dividing the $48.7 \mathrm{ft}(14.8 \mathrm{~m})$ long pipe into multiple conduits within the SWMM5 model. 
While making the numerous SWMM5 simulations, it became apparent that the time step that provided the maximum surge height at the riser shaft was related to the number of divisions and cell length in the SWMM5 model. The optimized time step for use in SWMM5 is given below:

$$
\Delta t=\frac{\Delta x}{a}
$$

where:

$$
\begin{aligned}
\Delta t & =\text { SWMM5 optimized time step } \\
\Delta x & =\text { divided conduit length }, \text { and } \\
\mathrm{a} & =\text { closed conduit wave speed. }
\end{aligned}
$$

The closed conduit wave speed may be calculated with the following equation:

$$
a=\sqrt{g \frac{A_{\text {full }}}{T_{\text {slot }}}}
$$

where:

$$
\begin{aligned}
a & =\text { wave speed } \\
g & =\text { gravitational constant } \\
\mathrm{A}_{\text {full }} & =\text { full pipe cross-sectional area, and } \\
\mathrm{T}_{\text {slot }} & =\text { Preissmann slot width. }
\end{aligned}
$$

The pipe was divided into 32, 158 and 300 smaller segments with connecting junctions at each end. The junctions that were added had very low and equivalent total surface areas. The time step in the SWMM5 model runs was then set to very low and optimized values using Equations 10.1 and 10.2 .

With the pipe divided into smaller segments and the time step optimized, much more accurate results were possible using SWMM5. A comparison of the physical model results to a number of SWMM5 model runs using conduit divisions with the optimized time step is presented in Figure 10.4.

While the results provided by SWMM5 after conduit division seem to be promising, it is important to evaluate the affect of the time step on the results. Because most sewer systems contain multiple pipes of varying sizes, it will not always be possible to select an optimized time step in SWMM5. Figure 10.5 shows that the SWMM5 model results with the pipe divided into 158 segments while the time step was varied both higher and lower than the optimized time step $(\mathrm{dt}=0.004304 \mathrm{sec}$.). These results show significantly worse results with higher or lower time steps. 


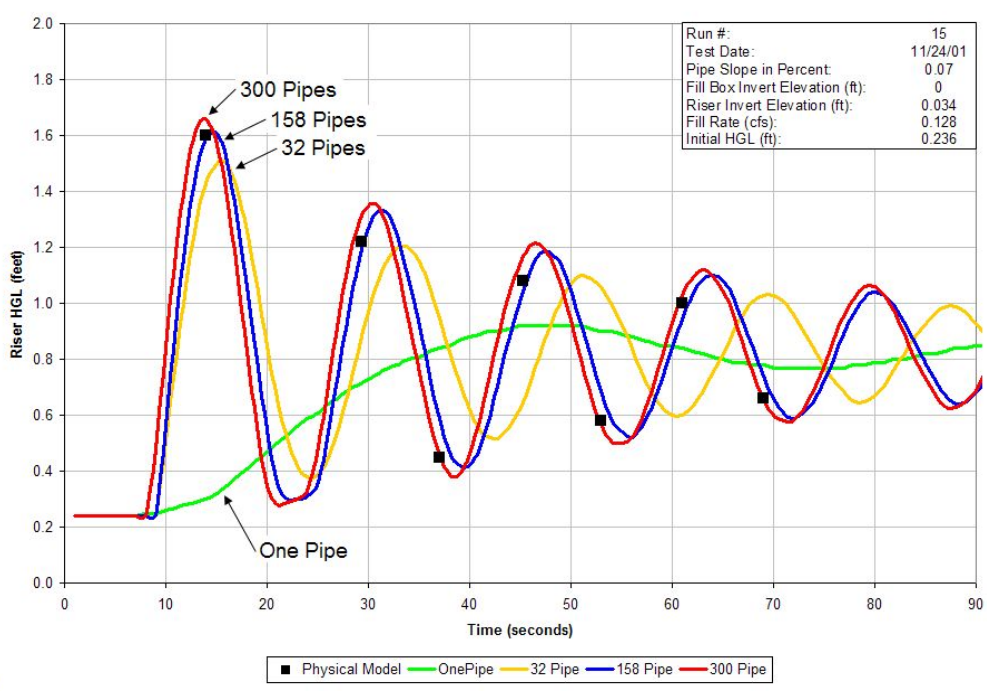

Figure 10.4 Comparison between physical model measurements and the SWMM5 divided conduit model simulation results.

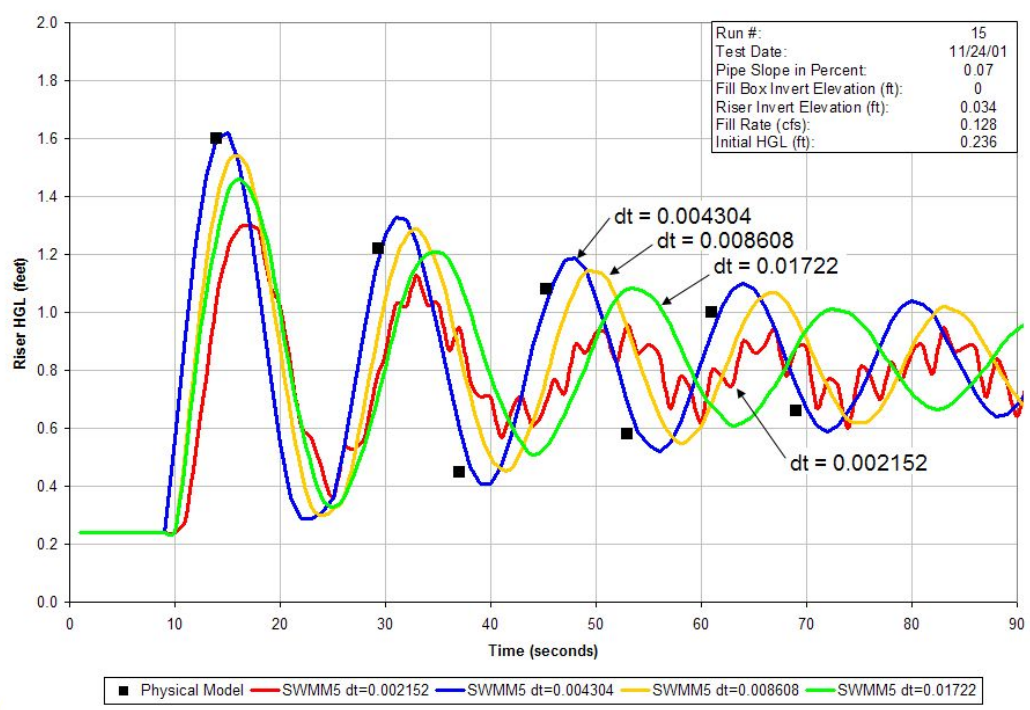

Figure 10.5 Comparison between physical model measurements and the SWMM5 simulation results with varying time step. 


\subsubsection{MOUSE and InfoWorks Results}

Both the MOUSE and InfoWorks software programs allowed the setup and simulation of the physical model conditions. However the model results were highly damped. These software programs are generally applied to large sewer systems to simulate steady-state or gradually varied flow conditions. Both models employ large Preissmann slot widths, which results in a reduced wave speed and over damping of the hydraulic bore, as well as nonadjustable or minimum time steps of one second or more. Because of these limitations, neither were able to accurately simulate the hydraulic surge conditions created in the physical model runs.

Because of limitation of a minimum allowable time step of one second, MOUSE only predicted an initial surge wave HGL of $1.09 \mathrm{ft}(0.33 \mathrm{~m})$, much lower than the actual surge HGL of $1.60 \mathrm{ft}(0.48 \mathrm{~m})$. The large Preissmann slot in the InfoWorks model predicted an initial surge wave HGL of only $1.22 \mathrm{ft}(0.37 \mathrm{~m})$. A comparison of the MOUSE, InfoWorks and TAP simulation results are shown in Figure 10.6.

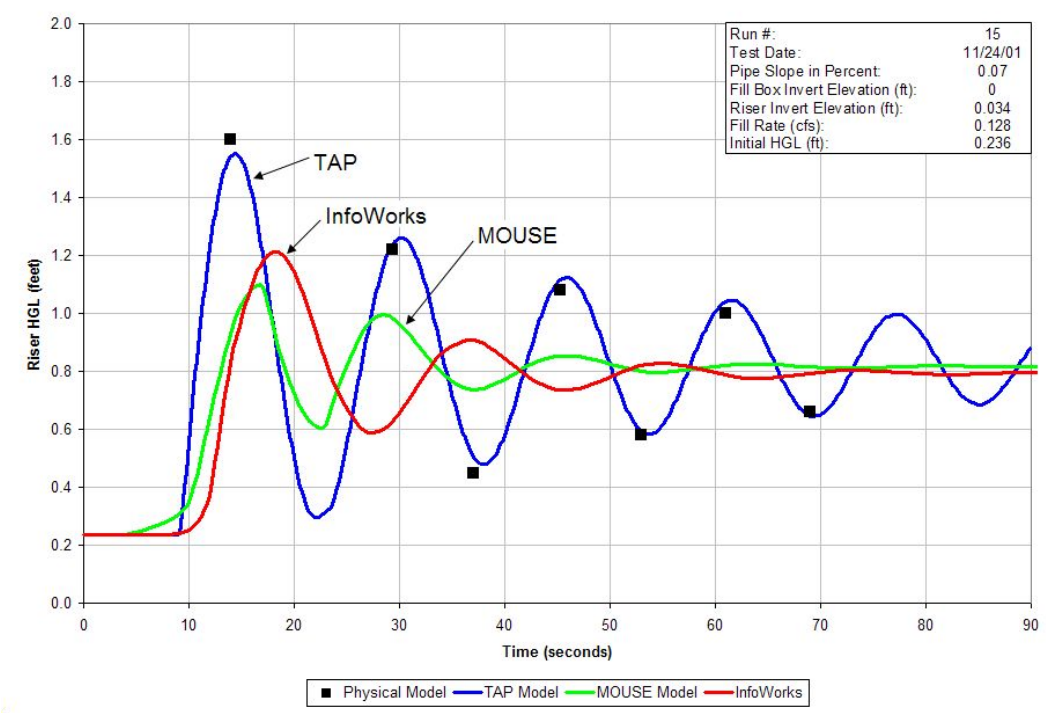

Figure 10.6 Comparison between physical model measurements and the MOUSE, InfoWorks and TAP simulation results. 


\subsection{Conclusions}

While many hydraulic modeling software programs may produce results with no indication of inaccuracy, the simulation results should still be scrutinized. This comparison shows the need for caution in evaluating hydraulic surge simulation results. Trusting results too quickly could result in the omission or the under designing of surge control facilities, resulting in damage to the sewer system and its associated structures, as well as environmental damages.

While MOUSE and InfoWorks produced simulation results, the predicted height of the surge had a high level of inaccuracy compared to the physical model results. SWMM5 was able to produce results comparable to the physical model results, but only after dividing the conduit into multiple sections and careful selection of time step. It is important to note that while this time step selection was possible for this model setup, real world simulations with multiple pipe sizes may prevent the selection of an optimized time step.

TAP provided accurate comparisons to the physical model with the least amount of setup issues. TAP automatically ensures that an optimized time step is used for every conduit in the simulation, and it also automatically divides each conduit into multiple reaches per user input. This automatic setup allows for easy and trustable simulation results.

\section{References}

Vasconcelos, J. G. 2005. Dynamic Approach to the Description of Flow Regime Transition in Stormwater Systems. PhD dissertation, Civil and Environmental Engineering Department, the University of Michigan.

Preissmann, A. 1961. "Propagation des intumescences dans les canaux et rivières", 1st Congress of the French Association for Computation (AFCALTI), September, Grenoble.

Crossley, A. J. 2003. "Local time stepping for modeling open channel flows." J. Hydraulic. Engineering., 129(6), 455-462.

Roe, P. L. 1981. "Approximate Riemann solvers, parameter vectors, and difference schemes." J. Comp. Physics, 43, 357-372.

Ridgway, K. and G.J. Kumpula. 2006. "Surge Modeling in Sewers using the Transient Analysis Program (TAP)." Journal of Water Management Modeling R227-06. doi: 10.14796/JWMM.R227-06. 